\section{Extensions to "Stability Analysis of Fuzzy Control Systems Subject to Uncertain Grades of Membership"}

\author{
Carlos Ariño and Antonio Sala, Member, IEEE
}

\begin{abstract}
In the December 2005 issue of this journal, Lam and Leung proposed stability results for fuzzy control systems where the membership functions in the controller were not the same as those from the process one, but some multiplicative bounds were known. The main practical context where that situation arises is the uncertain knowledge of the memberships of a Takagi-Sugeno model. This correspondence presents further extensions of those results, allowing for a richer description of the membership uncertainty, in terms of affine inequalities.
\end{abstract}

Index Terms-Fuzzy control, linear matrix inequalities (LMIs), parallel distributed compensation (PDC), quadratic stability, relaxed condition, Takagi-Sugeno (TS) fuzzy systems.

\section{INTRODUCTION}

Fuzzy control has reached maturity and acceptance nowadays via a formalization of the performance requirements and controller design techniques. In particular, there is a vast literature on control design for continuous, discrete, and delayed Takagi-Sugeno (TS) [2] fuzzy systems via linear matrix inequalities (LMIs) [3]-[7]. There may be other (possibilistic) interpretations of fuzziness in a control context [8]. The reader is referred to [9] and [10] for a review of the current trends and open issues in fuzzy modeling, identification, and control.

The majority of works on the fuzzy control for TS models assume the parallel distributed compensation (PDC) paradigm [3], i.e., the membership functions of the controller, e.g., $\eta_{i}$, are the same as the ones from the process, e.g., $\mu_{i}$. Furthermore, the proposed stability and performance conditions are shape-independent, i.e., valid for any membership function set conforming a fuzzy partition $\left(\mu_{i} \geq 0\right.$, $\left.\sum_{i} \mu_{i}=1\right)$.

Recent contributions in the non-PDC case $\left(\eta_{i} \neq \mu_{i}\right)$ are [1], [11], and [12]. In particular, in [1], LMI stability conditions were given for non-PDC fuzzy systems with uncertain degrees of membership expressed as a multiplicative uncertainty inequality $\rho_{i}^{m} \mu_{i} \leq \eta_{i} \leq \rho_{i}^{M} \mu_{i}$. Lam and Leung's conditions are shape-dependent in the sense that they achieve a reduction of conservativeness by setting up conditions which are only valid for membership functions having a constrained shape. In the same spirit, we [13], [14] present some shape-dependent conditions for the PDC case.

The main objective of this correspondence is to present shapedependent LMI conditions to design controllers for the TS fuzzy systems with uncertain memberships. The allowed uncertainty description is more general than that in [1], which did consider only the multiplicative uncertainty; such setup will be cast as a particular case of the one proposed here.

The structure of this correspondence is as follows. Section II describes the fuzzy systems and closed-loop equations to be discussed. Section III presents the main result which extends the uncertainty descriptions in literature. Section IV applies it to particular cases of additive and multiplicative uncertainties. Section V will show numeri-

Manuscript received March 5, 2007; revised August 24, 2007. This paper was recommended by Associate Editor S. X. Yang.

C. Ariño is with the Department of Industrial Systems Engineering and Design, Universitat Jaume I, 12071 Castelló de la Plana, Spain (e-mail: arino@esid.uji.es).

A. Sala is with the Department of Systems Engineering and Control, Technical University of Valencia, 46022 Valencia, Spain (e-mail: asala@isa.upv.es).

Digital Object Identifier 10.1109/TSMCB.2007.913596 cal examples illustrating the possibilities of the approach. A conclusion section closes this correspondence.

\section{Preliminaries AND Notation}

Stability of Closed-Loop TS Fuzzy Systems: Consider a TS fuzzy system [2] of order $n$ with $r$ rules

$$
\dot{x}=\sum_{i=1}^{r} \mu_{i}(z)\left(A_{i} x+B_{i} u\right) \quad \sum_{i=1}^{r} \mu_{i}(z)=1, \mu_{i}(z) \geq 0 .
$$

The aforementioned fuzzy system will be controlled via a statefeedback fuzzy controller (possibly non-PDC, where $\eta_{i}$ is a membership function that is different to $\mu_{i}$, at least in principle)

$$
u=-\sum_{i=1}^{r} \eta_{i}\left(z^{\prime}\right) F_{i} x \quad \sum_{i=1}^{r} \eta_{i}\left(z^{\prime}\right)=1, \eta_{i}\left(z^{\prime}\right) \geq 0
$$

where $z^{\prime}$ denotes measurable scheduling variables (possibly coincident with $z$ ). The controller yields a closed-loop [1]

$$
\dot{x}=\sum_{i=1}^{r} \sum_{j=1}^{r} \mu_{i}(z) \eta_{j}\left(z^{\prime}\right)\left(A_{i}-B_{i} F_{j}\right) x
$$

In the following, $\mu_{i}$ and $\eta_{j}$ will be used as shorthands for $\mu_{i}(z)$ and $\eta_{j}\left(z^{\prime}\right)$, respectively.

By considering a Lyapunov function $V(x)=x^{\mathrm{T}} P x, P>0$, it is straightforward to prove that (3) is a stable system with a decay rate $\alpha$ (i.e., $\|x\| \leq M e^{-\alpha t}$ for some $\left.M\right)$ if $(d V / d t)+2 \alpha V \leq 0$ for nonzero $x$ [3]. Such inequality, in the case of (3), amounts to the wellknown expression [1], [3]

$$
\begin{aligned}
-\left(\frac{d V}{d t}\right. & +2 \alpha V)=-x^{\mathrm{T}} \\
& \times\left(\sum_{i=1}^{r} \sum_{j=1}^{r} \mu_{i} \eta_{j} G_{i j}^{\mathrm{T}} P+P G_{i j}+2 \alpha P\right) x \geq 0 \quad \forall x \neq 0
\end{aligned}
$$

where $G_{i j}=A_{i}-B_{i} F_{j}$.

General Case: In a general case, many conditions for stability and performance of the closed-loop system (3) may be cast as positivity of fuzzy summations in the form

$$
\psi^{\mathrm{T}} \Theta \psi=\psi^{\mathrm{T}}\left(\sum_{i=1}^{r} \sum_{j=1}^{r} \mu_{i} \eta_{j} Q_{i j}\right) \psi \geq 0 \quad \forall \psi \neq 0
$$

where $Q_{i j}$ is a symmetric $\mathbb{R}^{n \times n}$ matrix, possibly including unknown decision variables to be found via optimization algorithms (usually LMI [3], [15]). For instance, (4) is a particular case of (5) with $\psi=x$ and

$$
Q_{i j}=-\left(\left(A_{i}-B_{i} F_{j}\right)^{\mathrm{T}} P+P\left(A_{i}-B_{i} F_{j}\right)+2 \alpha P\right) .
$$

As another example of a performance-related condition from [16], which is later used in Section V, we have

$$
Q_{i j}=\left(\begin{array}{ccc}
\Xi_{11} & B_{1 i} & P C_{i}^{\mathrm{T}}+R_{j}^{\mathrm{T}} D_{12 i}^{\mathrm{T}} \\
B_{1 i}^{\mathrm{T}} & -\gamma I & D_{11 i}^{\mathrm{T}} \\
C_{i} P+D_{12 i} R_{j} & D_{11 i} & -\gamma I
\end{array}\right)
$$


with $\Xi_{11}=P A_{i}^{\mathrm{T}}+R_{j}^{\mathrm{T}} B_{2 i}^{\mathrm{T}}+A_{i} P+B_{2 i} R_{j}$, which may be used to prove that there exists a stabilizing state-feedback controller such that the $H_{\infty}$ norm (i.e., $\mathcal{L}_{2}$-to- $\mathcal{L}_{2}$ induced norm) of a TS fuzzy system given by

$$
\begin{aligned}
& \dot{x}=\sum_{i=1}^{r} \mu_{i}(z)\left(A_{i} x+B_{1 i} v+B_{2 i} u\right) \\
& y=\sum_{i=1}^{r} \mu_{i}(z)\left(C_{i} x+D_{11 i} v+D_{12 i} u\right)
\end{aligned}
$$

is lower than $\gamma$. In [3], [9], [10], [12], and [13] other expressions of $Q_{i j}$ for continuous and discrete TS systems are considered.

Remark: Most of the cited literature considers $Q_{i j}$ for cases where $\eta_{i}=\mu_{i}$, i.e., PDC controllers $u=-\sum \mu_{i} F_{i} x$ with a closed loop $\dot{x}=\sum_{i=1}^{r} \sum_{j=1}^{r} \mu_{i}(z) \mu_{j}(z) x^{\mathrm{T}}\left(A_{i}-B_{i} F_{j}\right) x$, i.e., resulting in conditions such as

$$
\psi^{\mathrm{T}}\left(\sum_{i=1}^{r} \sum_{j=1}^{r} \mu_{i} \mu_{j} Q_{i j}\right) \psi \geq 0 \quad \forall \psi \neq 0
$$

where the only difference with (5) is that $\mu_{i} \mu_{j}$ appears instead of $\mu_{i} \eta_{j}$. In order to check the stability and performance of the non-PDC loop (3), it is straightforward to prove that, in most cases, the $Q_{i j}$ proposed in PDC literature appears unaltered in (5).

The difference between the PDC and the non-PDC cases lies in the LMIs needed to prove (5), which may be different to those needed for (10).

1) Indeed, in the PDC case (10), widely used sufficient conditions are the adaptation of [4, Th. 2], which may be generalized to a family of asymptotically necessary and sufficient ones [7].

2) In the non-PDC case (5), if $\mu_{i}$ and $\eta_{j}$ may be arbitrary, (5) holds if and only if the shape-independent conditions

$$
Q_{i j}>0 \quad \forall i, j
$$

do as, for instance, $\mu_{3}=\eta_{5}=1$ (the rest being zero) involves $\Theta=Q_{35}$ in (5) and the numbers three and five may be arbitrarily replaced by any $i$ or $j$.

Of course, all LMIs proving (5) - such as the ones in this correspondence-prove (10) as well, but they may be very conservative for the latter case, as numerical examples will show: that is the price to pay for imperfect knowledge of the plant memberships.

Shape-Dependent Cases: There are intermediate cases where the membership functions $\mu_{i}$ are not perfectly known (i.e., $\eta_{i} \neq \mu_{i}$ yielding non-PDC setups) but some knowledge on them is available. In that case, $\eta_{i}$ may be intentionally designed to be as similar as possible to $\mu_{i}$, and conditions which are less conservative than (11) may be stated. Indeed, Lam and Leung [1] state improved shape-dependent conditions which guarantee closed-loop stability ${ }^{1}$ of (3) when

$$
\rho_{i}^{m} \leq \frac{\eta_{i}}{\mu_{i}} \leq \rho_{i}^{M}
$$

given known values of the bounds $\rho_{i}^{m}$ and $\rho_{i}^{M}$.

The following lemma is the generalization of that in [1] removing the need of a symmetric $X_{i j}$, requiring only $X_{j i}=X_{i j}^{\mathrm{T}}$, and replacing $2 X_{i j}$ by $X_{i j}+X_{j i}$, in the same way as Liu and Zhang [4] generalize [17]. Details of the proof are omitted for brevity.

\footnotetext{
${ }^{1}$ The cited work discussed only one particular case of $Q_{i j}$, but it is, trivially, generalizable to any other performance-related expression for $Q_{i j}$.
}

Lemma 1: Given the bounds (12), (5) holds if there exist $P>0$ and matrices $X_{j i}=X_{i j}^{\mathrm{T}}$ such that

$$
\begin{aligned}
& \rho_{i}^{M} Q_{i i}-X_{i i}>0, \quad \rho_{i}^{m} Q_{i i}-X_{i i}>0 \\
& \rho_{j}^{M} Q_{i j}+\rho_{i}^{M} Q_{j i}-\left(X_{i j}+X_{j i}\right)>0 \\
& \rho_{j}^{m} Q_{i j}+\rho_{i}^{m} Q_{j i}-\left(X_{i j}+X_{j i}\right)>0 \\
& \rho_{j}^{m} Q_{i j}+\rho_{i}^{M} Q_{j i}-\left(X_{i j}+X_{j i}\right)>0 \\
& \rho_{j}^{M} Q_{i j}+\rho_{i}^{m} Q_{j i}-\left(X_{i j}+X_{j i}\right)>0 \\
& \left(\begin{array}{ccc}
X_{11} & \ldots & X_{1 r} \\
\vdots & \ddots & \vdots \\
X_{r 1} & \ldots & X_{r r}
\end{array}\right)>0 .
\end{aligned}
$$

The developments in the next section will present additional results for a more general class of constraints than the "multiplicative uncertainty" (12). Numerical examples in Section V will show that, apart from allowing for more general uncertainty descriptions, the new conditions provide better results than those in [1] with multiplicative uncertainty, at least in some cases.

\section{MAIN RESUlT}

Consider a set of $p$ constraints on the shape of the membership functions of the plant $\mu_{i}$ and the controller $\eta_{i}$ given by the affine inequalities

$$
\mathbf{c}_{k}^{\mathrm{T}} \eta+\mathbf{a}_{k}^{\mathrm{T}} \mu+b_{k} \leq 0, \quad k=1, \ldots, p
$$

where $\eta$ and $\mu$ denote the membership functions arranged as a column vector, $\mathbf{c}_{k}$ and $\mathbf{a}_{k}$ are also column vectors, and $b_{k}$ is a scalar. $\mathbf{c}_{k}, \mathbf{a}_{k}$, and $b_{k}$ are assumed to be known. Notations $a_{i k}$ and $c_{i k}$ will denote the $i$ th component of vectors $\mathbf{a}_{k}$ and $\mathbf{c}_{k}$, respectively. For instance, the constraint $\mu_{2}+\mu_{1} \leq 2 \eta_{1}+0.05$, in a three-rule fuzzy system, is trivially expressed in (19) with $\mathbf{c}=\left(\begin{array}{lll}-2 & 0 & 0\end{array}\right)^{\mathrm{T}}, \mathbf{a}=\left(\begin{array}{lll}1 & 1 & 0\end{array}\right)^{\mathrm{T}}$, and $b=-0.05$. Constraint $\eta_{1} \leq 2 \mu_{1}$ (the particular case contemplated in [1]) requires $\mathbf{c}=\left(\begin{array}{lll}1 & 0 & 0\end{array}\right)^{\mathrm{T}}, \mathbf{a}=\left(\begin{array}{lll}-2 & 0 & 0\end{array}\right)^{\mathrm{T}}$, and $b=0$.

Theorem 1: If (19) is known to hold, (5) holds if there exist matrices $X_{i j}=X_{j i}^{\mathrm{T}}, i, j=1, \ldots, 2 r$, and symmetric definite positive matrices $R_{j k}$ and $R_{j k}^{\dagger}$ such that for all $j=1, \ldots, r$ and $k=1, \ldots, p$

$$
\begin{aligned}
& \sum_{k=1}^{p}\left(a_{j k} R_{i k}+a_{i k} R_{j k}\right) \geq X_{i j}+X_{j i} \\
& Q_{i j}+\sum_{k=1}^{p}\left(c_{j k} R_{i k}+a_{i k} R_{j k}^{\dagger}+b_{k}\left(R_{i k}+R_{j k}^{\dagger}\right)\right) \\
& \geq X_{i(j+r)}+X_{(j+r) i} \\
& \sum_{k=1}^{p}\left(\begin{array}{ccc}
\left.c_{i k} R_{j k}^{\dagger}+c_{j k} R_{i k}^{\dagger}\right) \geq X_{(I+r)(j+r)}+X_{(j+r)(i+r)} \\
X_{11} & \cdots & X_{1(2 r)} \\
\vdots & \ddots & \vdots \\
X_{(2 r) 1} & \cdots & X_{(2 r)(2 r)}
\end{array}\right)>0 .
\end{aligned}
$$

If $Q_{i j}$ is linear in some matrix decision variables, then Theorem 1 provides LMI conditions. 
Proof: Expression (19) may be written as

$$
\sum_{i=1}^{r} c_{i k} \eta_{i}+a_{i k} \mu_{i}+b_{k} \leq 0, \quad k=1, \ldots, p
$$

Consider now, for a particular fixed $k$, the matrix $\Gamma_{k}=$ $\sum_{j=1}^{r}\left(\mu_{j} R_{j k}+\eta_{j} R_{j k}^{\dagger}\right)$. Evidently, $\Gamma_{k} \geq 0$ because it is a sum with positive coefficients of positive definite matrices. For a particular $k$, multiplying (24) by $\Gamma_{k}$, we get

$$
\begin{aligned}
& \sum_{i=1}^{r} \sum_{j=1}^{r}\left(c_{i k} R_{j k} \eta_{i} \mu_{j}+c_{i k} R_{j k}^{\dagger} \eta_{i} \eta_{j}+a_{i k} R_{j k} \mu_{i} \mu_{j}\right. \\
& \left.\quad+a_{i k} R_{j k}^{\dagger} \mu_{i} \eta_{j}\right)+b_{k} \sum_{j=1}^{r}\left(\mu_{j} R_{j k}+\eta_{j} R_{j k}^{\dagger}\right) \leq 0
\end{aligned}
$$

Subsequently, by using the equalities $\sum_{i=1}^{r} \mu_{i}=1$ and $\sum_{i=1}^{r} \eta_{i}=1$ in

$$
b_{k} \Gamma_{k}=b_{k} \sum_{j=1}^{r}\left(\sum_{i=1}^{r} \eta_{i} \mu_{j} R_{j k}+\sum_{i=1}^{r} \mu_{i} \eta_{j} R_{j k}^{\dagger}\right)
$$

we get a negative-semidefinite matrix to be denoted as $H_{k}$ given by

$$
\begin{array}{r}
H_{k}=\sum_{i=1}^{r} \sum_{j=1}^{r}\left(c_{i k} R_{j k} \eta_{i} \mu_{j}+c_{i k} R_{j k}^{\dagger} \eta_{i} \eta_{j}+a_{i k} R_{j k} \mu_{i} \mu_{j}\right. \\
\left.\quad+a_{i k} R_{j k}^{\dagger} \mu_{i} \eta_{j}+b_{k}\left(\eta_{i} \mu_{j} R_{j k}+\mu_{i} \eta_{j} R_{j k}^{\dagger}\right)\right)
\end{array}
$$$$
\leq 0
$$

As (26) holds for each $k$, denoting by $H=\sum_{k=1}^{p} H_{k}$, evidently $H \leq 0$, i.e.,

$$
\begin{array}{r}
H=\sum_{k=1}^{p} \sum_{i=1}^{r} \sum_{j=1}^{r}\left(c_{i k} R_{j k} \eta_{i} \mu_{j}+c_{i k} R_{j k}^{\dagger} \eta_{i} \eta_{j}+a_{i k} R_{j k} \mu_{i} \mu_{j}\right. \\
\left.\quad+a_{i k} R_{j k}^{\dagger} \mu_{i} \eta_{j}+b_{k}\left(\eta_{i} \mu_{j} R_{j k}+\mu_{i} \eta_{j} R_{j k}^{\dagger}\right)\right)
\end{array}
$$$$
\leq 0
$$

By taking $H$ from the aforementioned equation and $\Theta$ from (5), it is evident that if $\Theta+H>0$ can be proved, then $\Theta>0$. Then, conveniently grouping terms

$$
\begin{array}{r}
\Theta+H \\
=\sum_{i=1}^{r} \sum_{j=1}^{r}\left(\mu_{i} \mu_{j}\left(\sum_{k=1}^{p} a_{i k} R_{j k}\right)+\eta_{i} \eta_{j}\left(\sum_{k=1}^{p} c_{i k} R_{j k}^{\dagger}\right)\right. \\
+\mu_{i} \eta_{j}\left(Q_{i j}+\sum_{k=1}^{p}\left(\begin{array}{c}
c_{j k} R_{i k}+a_{i k} R_{j k}^{\dagger} \\
\left.\left.\left.+b_{k}\left(R_{i k}+R_{j k}^{\dagger}\right)\right)\right)\right)
\end{array}\right.\right.
\end{array}
$$

$$
\begin{gathered}
=\sum_{i=1}^{r}\left(\mu_{i}^{2} \sum_{k=1}^{p} a_{i k} R_{i k}+\eta_{i}^{2} \sum_{k=1}^{p} c_{i k} R_{i k}^{\dagger}\right) \\
+\sum_{i<j \leq r}\left(\mu_{i} \mu_{j}\left(\sum_{k=1}^{p}\left(a_{i k} R_{j k}+a_{j k} R_{i k}\right)\right)\right. \\
\left.+\eta_{i} \eta_{j}\left(\sum_{k=1}^{p} c_{i k} R_{j k}^{\dagger}+c_{j k} R_{i k}^{\dagger}\right)\right) \\
+\sum_{i=1}^{r} \sum_{j=1}^{r} \mu_{i} \eta_{j}\left(Q_{i j}+\sum_{k=1}^{p}\left(c_{j k} R_{i k}+a_{i k} R_{j k}^{\dagger}\right.\right. \\
\left.\left.+b_{k}\left(R_{i k}+R_{j k}^{\dagger}\right)\right)\right) .
\end{gathered}
$$

Consider now the variables $X_{i j}=X_{j i}^{\mathrm{T}}, i, j=1, \ldots, r$, which fulfill (20)-(22). By suitably grouping terms and associating (20) to the terms where $\mu_{i} \mu_{j}$ appears, (21) to those with $\mu_{i} \eta_{j}$, and (22) to those with $\eta_{i} \eta_{j}$, we have ${ }^{2}$

$$
\begin{aligned}
\Theta+H \geq & \sum_{i=1}^{r}\left(\mu_{i}^{2} X_{i i}+\eta_{i}^{2} X_{i i}\right) \\
& +\sum_{i=1}^{r} \sum_{i<j \leq r}\left(\mu_{i} \mu_{j}\left(X_{i j}+X_{j i}\right)+\eta_{i} \eta_{j}\right. \\
& \left.\times\left(X_{(i+r)(j+r)}+X_{(j+r)(i+r)}\right)\right) \\
& +\sum_{i=1}^{r} \sum_{j=1}^{r}\left(\mu_{i} \eta_{j}\left(X_{i(j+r)}+X_{(j+r) i}\right)\right) .
\end{aligned}
$$

Considering now the original fuzzy summations of matrix $\Theta$ in (5) and defining

$$
\xi=\mu \otimes \psi=\left[\begin{array}{lllll}
\mu_{1} \psi^{\mathrm{T}}, & \ldots, & \mu_{r} \psi^{\mathrm{T}} \quad \eta_{1} \psi^{\mathrm{T}}, \quad \ldots, \quad \eta_{r} \psi^{\mathrm{T}}
\end{array}\right]^{\mathrm{T}}
$$

we have, from (29)

$$
\psi^{\mathrm{T}} \Theta \psi \geq \psi^{\mathrm{T}}(\Theta+H) \psi \geq \xi^{\mathrm{T}}\left(\begin{array}{ccc}
X_{11} & \cdots & X_{1(2 r)} \\
\vdots & \ddots & \vdots \\
X_{(2 r) 1} & \cdots & X_{(2 r)(2 r)}
\end{array}\right) \xi .
$$

Hence, if (23) holds, $\psi^{\mathrm{T}}(\Theta+H) \psi>0$ for $\psi \neq 0$, and therefore, (5) holds.

\section{Particular Cases}

Let us now consider some particular cases of Theorem 1 .

\section{A. Multiplicative Uncertainty}

Consider now the multiplicative uncertainty case, which is also discussed in [1]

$$
\rho_{i}^{m} \leq \frac{\eta_{i}}{\mu_{i}} \leq \rho_{i}^{M}
$$

${ }^{2}$ Note that (20) implies $\sum_{k=1}^{p} a_{i k} R_{i k} \geq X_{i i}$, and an analogous consideration may be made with (22): the diagonal terms $\mu_{i}^{2}$ and $\eta_{i}^{2}$ need not be explicitly written in the theorem statement. 
Corollary 1: If (30) is known to hold, (5) holds if there exist matrices $X_{i j}=X_{j i}^{\mathrm{T}}, i, j=1, \ldots, 2 r$, and symmetric definite positive matrices $R_{i j}, N_{j i}, R_{i j}^{\dagger}$, and $N_{j i}^{\dagger}$ such that for all $i, j=1, \ldots, r$

$$
\begin{aligned}
& R_{i j} \rho_{j}^{m}-N_{i j} \rho_{j}^{M}+R_{j i} \rho_{i}^{m}-N_{j i} \rho_{i}^{M} \geq X_{i j}+X_{j i} \\
& Q_{i j}-\left(R_{i j}-N_{i j}\right)-\left(R_{i j}^{\dagger}-N_{i j}^{\dagger}\right) \geq X_{i(j+r)}+X_{(j+r) i} \\
& \frac{R_{i j}^{\dagger}}{\rho_{i}^{M}}-\frac{N_{i j}^{\dagger}}{\rho_{i}^{m}}+\frac{R_{j i}^{\dagger}}{\rho_{j}^{M}}-\frac{N_{j i}^{\dagger}}{\rho_{j}^{m}} \geq X_{(i+r)(j+r)}+X_{(j+r)(i+r)} \\
& \left(\begin{array}{ccc}
X_{11} & \cdots & X_{1(2 r)} \\
\vdots & \ddots & \vdots \\
X_{(2 r) 1} & \cdots & X_{(2 r)(2 r)}
\end{array}\right)>0 .
\end{aligned}
$$

Proof: The uncertainty description can be expressed as

$$
\eta_{k}-\rho_{k}^{M} \mu_{k} \leq 0 \quad-\eta_{k}+\rho_{k}^{m} \mu_{k} \leq 0
$$

Hence, the theorem will be proved by using Theorem 1 with $2 r$ constraints, which is divided into two groups (both with $b_{k}=0$ ).

1) One with $a_{k k}=-\rho_{k}^{M}, a_{i k}=0$ for $i \neq k$ and $c_{k k}=+1$, $c_{i k}=0$ for $i \neq k$; Theorem 1 will be applied using $M_{i k}$ and $M_{i k}^{\dagger}$ as relaxation variables.

2) Another group (consider $a_{k}^{\prime}=a_{k+r}, c_{k}^{\prime}=c_{k+r}$ ) with $a_{k k}^{\prime}=$ $\rho_{k}^{m}, a_{i k}^{\prime}=0$ for $i \neq k$ and $c_{k k}^{\prime}=-1, c_{i k}^{\prime}=0$ for $i \neq k$; Theorem 1 will be applied using $T_{i k}$ and $T_{i k}^{\dagger}$ as relaxation variables.

Note that (20)-(22) in this case reduce to

$a_{j j} M_{i j}+a_{i i} M_{j i}+a_{j j}^{\prime} T_{i j}+a_{i i}^{\prime} T_{j i} \geq X_{i j}+X_{j i}$
$Q_{i j}+\left(c_{j j} M_{i j}+a_{i i} M_{j i}^{\dagger}+c_{j j}^{\prime} T_{i j}+a_{i i}^{\prime} T_{j i}^{\dagger}\right) \geq X_{i(j+r)}+X_{(j+r) i}$

$c_{i i} M_{j i}^{\dagger}+c_{j j} M_{i j}^{\dagger}+c_{i i}^{\prime} T_{j i}^{\dagger}+c_{j j}^{\prime} T_{i j}^{\dagger} \geq X_{(i+r)(j+r)}+X_{(j+r)(i+r)}$

because $a_{i k}, c_{i k}, a_{i k}^{\prime}$, and $c_{i k}^{\prime}$ are zero for $i \neq k$.

The conditions for the theorem being proved arise immediately once the aforementioned particular values of $a_{i k}, c_{i k}, a_{i k}^{\prime}$, and $c_{i k}^{\prime}$ are substituted in (36)-(38), and the following changes of variable are made:

$$
M_{i j}=N_{i j} \quad T_{i j}=R_{i j} \quad \rho_{i}^{M} M_{i j}^{\dagger}=R_{i j}^{\dagger} \quad \rho_{i}^{m} T_{i j}^{\dagger}=N_{i j}^{\dagger}
$$

\section{B. Additive Uncertainty}

Consider a set of known additive bounds on the membership function $\delta_{k}$ so that, given (5), it is known that

$$
\left|\mu_{k}-\eta_{k}\right| \leq \delta_{k}, \quad k=1, \ldots, r .
$$

Corollary 2: If the membership functions satisfy (39), (5) holds if there exist matrices $R_{i j}, N_{i j}, X_{i j}=X_{j i}^{\mathrm{T}}$, and $X_{i(j+r)}=$ $X_{(j+r) i}^{\mathrm{T}}, i, j=1, \ldots, r$, such that

$$
\begin{aligned}
M_{i j}=R_{i j}-N_{i j} \quad M_{i j}^{+} & =R_{i j}+N_{i j} \\
M_{i j}+M_{j i} & \geq X_{i j}+X_{j i} \\
Q_{i j}-2 M_{i j}-\sum_{k=1}^{r} \delta_{k}\left(M_{i k}^{+}+M_{k j}^{+}\right) & \geq X_{i(j+r)}+X_{(j+r) i}
\end{aligned}
$$

for all $i=1, \ldots, r$ and $j=1, \ldots, r$ and

$$
\begin{aligned}
Y_{11} & =\left(\begin{array}{ccc}
X_{11} & \ldots & X_{1 r} \\
\vdots & \ddots & \vdots \\
X_{r 1} & \ldots & X_{r r}
\end{array}\right) \\
Y_{12} & =\left(\begin{array}{ccc}
X_{1(r+1)} & \ldots & X_{1(2 r)} \\
\vdots & \ddots & \vdots \\
X_{r(r+1)} & \ldots & X_{r(2 r)}
\end{array}\right) \quad\left(\begin{array}{cc}
Y_{11} & Y_{12} \\
Y_{12}^{\mathrm{T}} & Y_{11}
\end{array}\right)>0 .
\end{aligned}
$$

Proof: The uncertainty description can be expressed as

$$
\eta_{k}-\mu_{k}-\delta_{k} \leq 0 \quad-\eta_{k}+\mu_{k}-\delta_{k} \leq 0 .
$$

Hence, the theorem will be proved by using Theorem 1 with $2 r$ constraints, which is divided into two groups.

1) One with $b_{k}=-\delta_{k}, a_{k k}=1, a_{i k}=0$ for $i \neq k$ and $c_{k k}=-1$, $c_{i k}=0$ for $i \neq k$; Theorem 1 will be applied using $R_{i k}$ and $R_{i k}^{\dagger}$ as relaxation variables.

2) Another one with $b_{k}^{\prime}=-\delta_{k}, a_{k k}^{\prime}=-1, a_{i k}^{\prime}=0$ for $i \neq k$ and $c_{k k}^{\prime}=+1, c_{i k}^{\prime}=0$ for $i \neq k$; Theorem 1 will be applied using $N_{i k}$ and $N_{i k}^{\dagger}$ as relaxation variables.

By noting that most $a_{i k}$ 's and $c_{i k}$ 's are zero and defining $a_{i(k+r)}=$ $a_{i k}^{\prime}$, etc., Theorem 1 results in

$$
\begin{aligned}
& R_{i j}+R_{j i}-N_{i j}-N_{j i} \geq X_{i j}+X_{j i} \\
& Q_{i j}+\left(-R_{i j}+R_{j i}^{\dagger}+N_{i j}-N_{j i}^{\dagger}\right) \\
& \quad+\sum_{k=1}^{r}\left(-\delta_{k}\left(R_{i k}+R_{j k}^{\dagger}+N_{i k}+N_{j k}^{\dagger}\right)\right) \\
& \quad \geq X_{i(j+r)}+X_{(j+r) i} \\
& -R_{j i}^{\dagger}-R_{i j}^{\dagger}+N_{j i}^{\dagger}+N_{i j}^{\dagger} \\
& \quad \geq X_{(i+r)(j+r)}+X_{(j+r)(i+r)}
\end{aligned}
$$

and the corollary results once the following equalities are enforced: $R_{i j}^{\dagger}=N_{j i}$ and $N_{i j}^{\dagger}=R_{j i}$.

The next two lemmas show the following: 1) the PDC case is recovered from the above corollaries under no uncertainty; and 2) as expected, the conditions proposed with the additive or multiplicative uncertainty bounds are less conservative than the trivial ones $Q_{i j}>0$.

Lemma 2: When the membership error bound $\delta_{i}$ is equal to zero or the multiplicative bounds are equal to one (i.e., $\mu_{i}=\eta_{i}$ ), a feasible set of variables for Corollaries 1 and 2 may be obtained if [4, Th. 2] (which applies to the PDC case) is feasible.

Proof: Note first that, when $\delta=0$ and $\rho_{i}^{m}=\rho_{i}^{M}=1$, enforcing $R_{i j}^{\dagger}=R_{i j}, N_{i j}^{\dagger}=N_{i j}$, and $X_{(i+r)(j+r)}=X_{i j}$ in Corollary 1 leaves (31)-(34) identical to (41)-(43) in Corollary 2; thus, a unified analysis is possible, considering only Corollary 2 in the sequel.

If $\delta_{i}=0$ for all $i$ 's, then (42) can be rewritten as

$$
Q_{i j}-2 M_{i j} \geq X_{i(j+r)}+X_{(j+r) i} .
$$

By taking $M_{i j}=Q_{i j} / 2$ and $X_{i(j+r)}=0$, which fulfill (48), the inequality (41) gets converted into

$$
Q_{i j} / 2+Q_{j i} / 2 \geq X_{i j}+X_{j i}
$$

and the matrix $Y_{12}$ is equal to zero. Finally, (43) is

$$
\left(\begin{array}{cc}
Y_{11} & 0 \\
0 & Y_{11}
\end{array}\right)>0
$$


TABLE I

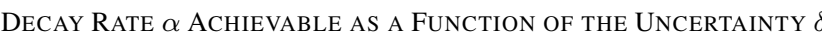

\begin{tabular}{|c||c|c|c|c|c|c|c|c|}
\hline$\delta$ & $0(\mathrm{PDC})$ & 0.05 & 0.1 & 0.15 & 0.2 & 0.25 & 0.3 & 1 (linear) \\
\hline$\alpha$ & 0.36 & 0.35 & 0.33 & 0.31 & 0.30 & 0.29 & 0.28 & 0.28 \\
\hline
\end{tabular}

equivalent to $Y_{11}>0$. This condition and (49) are the ones in [4, Th. 2]: if the latter is feasible, Corollaries 1 and 2 without uncertainty will be feasible as well.

Lemma 3: If $Q_{i j}>0$ for all $i, j=1, \ldots, r$, then Corollaries 1 and 2 are satisfied.

Proof: Regarding Corollary 2, take all $N_{i j}=0$ and all $X_{(i+r) j}=X_{i(j+r)}=0$. Then, take $R_{i j}=0$ for $i \neq j$ and $R_{i i}=\epsilon_{i} I$ for $i=1, \ldots, r$, choosing a small enough $\epsilon_{i}>0$ so that $Q_{i i} \gg 2 \epsilon_{i} I$ ( $I$ denotes the identity matrix). Then, $X_{i j}=0, i \neq j$, fulfills (41) and (42), and for $i=j, X_{i i}=\epsilon_{i} I$ fulfills (41). Finally, all $X_{i j}$ 's form a diagonal positive matrix that satisfies (43) if $\epsilon$ is small enough.

Corollary 1 is also satisfied with the same choice of $N_{i j}$ and $R_{i j}$ plus $N_{i j}^{\dagger}=N_{i j}$ and $R_{i j}^{\dagger}=R_{i j}$. Details are analogous to those above for Corollary 2.

\section{EXAMPLES}

This section presents several examples which illustrate the possibilities of the methodology with the additive and multiplicative uncertainties, in decay-rate and $\mathcal{H}_{\infty}$ settings.

Example 1: Let us consider a three-rule TS system $\dot{x}=$ $\sum_{i=1}^{3} \mu_{i}(x)\left(A_{i} x+B_{i} u\right)$ where

$$
\begin{array}{rlrl}
A_{1} & =\left(\begin{array}{ccc}
0.39 & 0.85 & 0.48 \\
0.81 & 0.010 & 0.34 \\
0.51 & 0.28 & 0.078
\end{array}\right) & B_{1}=\left(\begin{array}{cc}
0.1 & 0.58 \\
0.016 & 0.32 \\
0.80 & 0.58
\end{array}\right) \\
A_{2}=\left(\begin{array}{ccc}
0.0089 & 0.35 & 0.96 \\
0.76 & 0.54 & 0.38 \\
0.14 & 0.85 & 0.25
\end{array}\right) & B_{2}=\left(\begin{array}{cc}
0.031 & 0.036 \\
0.87 & 0.53 \\
0.75 & 0.78
\end{array}\right) \\
A_{3}=\left(\begin{array}{ccc}
0.84 & 0.094 & 0.8 \\
0.19 & 0.2 & 0.13 \\
0.82 & 0.58 & 0.33
\end{array}\right) & B_{3}=\left(\begin{array}{cc}
0.054 & 0.16 \\
0.21 & 0.84 \\
0.47 & 0.64
\end{array}\right) .
\end{array}
$$

A state-feedback fuzzy controller with the structure

$$
u=-\sum_{j=1}^{3} \eta_{j}(x) F_{j} x
$$

is proposed, where function $\eta_{j}(x)$ is an approximation of $\mu_{j}(x)$ fulfilling (39), for a shared $\delta_{k}=\delta$. Several values of the uncertainty $\delta$ will be tested, ranging from $\delta=0$ (which is the well-known PDC case $\eta_{i}=\mu_{i}$ ) to $\delta=1$ (indicating an absolute ignorance on the shape of $\mu_{i}$ ).

The control objective will be to find the $F_{j}$ maximizing the achievable quadratic decay rate $\alpha$ by checking (5) with

$$
Q_{i j}=-A_{i} Y-Y A_{i}^{\mathrm{T}}+B_{i} M_{j}+M_{j}^{\mathrm{T}} B_{i}-2 \alpha Y
$$

arising from (4) with the usual change of variables $Y=P^{-1}$ and $M_{j}=F_{j} Y$. The sufficient conditions provided in Corollary 2 will be used, searching for the maximum value of $\alpha$ for which a feasible LMI solution exists. The maximum $\alpha$ achieved for different values of $\delta$ appears in Table I.

The results in the referred table show that the more precise the knowledge of $\mu$ is (i.e., the lower $\delta$ is), the faster decay rates can be achieved. The results for $\delta=0$ are coincident with those [4, Th. 2] for the PDC case, as discussed in Lemma 2. Furthermore, the results for $\delta=1$ are coincident with the ones obtained by using a plain, nonfuzzy, and linear regulator $u=-K x$ robustly stabilizing a polytopic system via the LMI conditions [15]

$$
Q_{i}=-A_{i} Y-Y A_{i}^{\mathrm{T}}+B_{i} M+M^{\mathrm{T}} B_{i}-2 \alpha Y>0 .
$$

Such conditions are, in fact, equivalent to the shape-independent ones $Q_{i j}>0$ (indeed, conditions for $M_{1}$ in (51) are the same as those for $M_{2}$, etc.; therefore, there is no loss of generality by assuming that $M_{1}=M_{2}=\cdots=M$ ).

In summary, with the methodology in this correspondence, a smooth transition between a full-PDC fuzzy controller and a robust linear one is achieved: as uncertainty increases, the feasible performance decreases. If the uncertainty in memberships is greater than 0.3 , the performance of fuzzy and nonfuzzy (i.e., plain linear) controllers is the same. For lower uncertainty levels, the fuzzy control outperforms linear regulators, as expected.

The aforementioned model has also been tested with the multiplicative uncertainty bounds. In particular, with $\rho_{j}^{M}=3$ and $\rho_{j}^{m}=1 / 3$, application of the procedure in [1] yields an achievable decay rate of 0.304, application of Lemma 1 yields a decay rate of 0.308 (i.e., the additional decision variables achieve a marginal improvement), and Corollary 1 produces the best result, proving that a decay 0.322 is achievable.

Example 2: The same model from the previous example, with the multiplicative uncertainty bounds $\rho_{j}^{M}=3$ and $\rho_{j}^{m}=1 / 3$, has been used for the state-feedback $\mathcal{H}_{\infty}$ control, with $Q_{i j}$ as given in (7). If a disturbance input with $B_{1 i}=\left(\begin{array}{lll}-1 & 1-1\end{array}\right)^{\mathrm{T}}$ is considered and an output $y=\left(\begin{array}{lll}0 & 1 & 0\end{array}\right) x$, the following results are obtained: 1$)$ a robust linear regulator obtains an induced-norm bound of $6.988 ; 2$ ) a full PDC results in 6.437 , i.e., a better disturbance rejection, as expected; and 3) uncertainty in memberships yields intermediate values, which are better than the linear controller but worse than the PDC one: 6.726 for the original result in [1], 6.699 for Lemma 1, and 6.593 for Corollary 1.

Example 3: Let us now discuss the same example as in [1] regarding the multiplicative uncertainty. Consider a TS fuzzy system with two rules, with matrices

$$
\begin{aligned}
& A_{1}=\left(\begin{array}{cc}
2 & -10 \\
1 & 0
\end{array}\right) \\
& A_{2}=\left(\begin{array}{cc}
a & -10 \\
1 & 1
\end{array}\right) \\
& B_{1}=\left(\begin{array}{l}
1 \\
0
\end{array}\right) \\
& B_{2}=\left(\begin{array}{l}
b \\
0
\end{array}\right) .
\end{aligned}
$$

Analogously to [1], a two-rule state-feedback fuzzy controller is built by designing the $F_{i}, i=1,2$, by pole placement so that the closed-loop poles of $A_{i}-B_{i} F_{i}$ are at -1 and -15 (unique solution). Then, stability of the overall closed-loop system (3) is tested for different values of $a$ and $b$ and different values of $\rho_{i}^{M}$ and $\rho_{i}^{m}$ in (30).

Fig. 1 shows the values of $a$ and $b$ for which the closed loop can be proved stable for different uncertainty levels: The left plot presents the results with Corollary 1 , whereas the right one presents the results ${ }^{3}$ with Lemma 1 ; the tested uncertainty values were $\rho_{i}^{M}=\epsilon$ and $\rho_{i}^{m}=1 / \epsilon$ for $\epsilon$ taking values in $\{2,1.5,1.2,1.1,1\}$. All points

\footnotetext{
${ }^{3}$ In this example, Lemma 1 obtained results which were coincident with those obtained using the original decision variables in [1]
} 

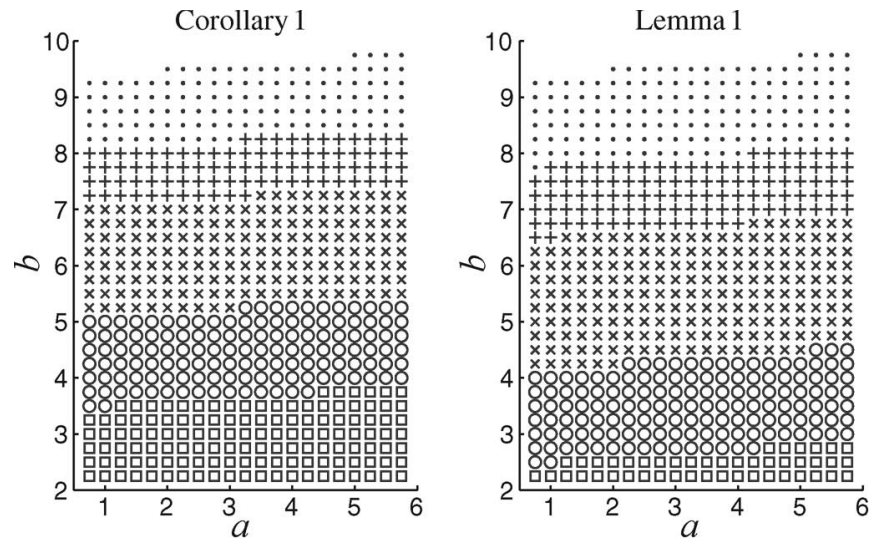

Fig. 1. Feasible values of parameters $a$ and $b$ for Example 2. (Left) Corollary 1 and (right) Lemma 1. Legend: [ $\square$ symbol] $: \rho_{i}^{M}=2, \rho_{i}^{m}=1 / 2 ;[\bigcirc$ symbol]: $\rho_{i}^{M}=1.5, \rho_{i}^{m}=1 / 1.5 ;\left[\times\right.$ symbol]: $\rho_{i}^{M}=1.2, \rho_{i}^{m}=1 / 1.2 ;[+$ symbol]: $\rho_{i}^{M}=1.1, \rho_{i}^{m}=1 / 1.1$; [• symbol]: $\rho_{i}^{M}=\rho_{i}^{m}=1$, i.e., PDC controller.

feasible for a particular value of $\epsilon$ were also feasible for lower values of it.

Clearly, Corollary 1 in this correspondence achieves better results than [1] in all tested uncertain cases, i.e., it finds a larger set of values for $a$ and $b$ yielding a stable closed loop. As expected, for no uncertainty in memberships $\left(\rho_{i}^{M}=\rho_{i}^{m}=1\right)$, the PDC case is recovered in both cases (denoted with $\bullet$ in the figure), with results coincident to those from [4, Th. 2].

\section{CONCLUSION}

This correspondence presents an extension of the methodology in [1] to consider arbitrary affine constraints in the shape of uncertain membership functions in a non-PDC fuzzy control setup. The proposed extensions apply to various stability and performance requirements in continuous and discrete systems by making different choices for $Q_{i j}$.

With the same type of restrictions than [1], numerical examples illustrate that performance improvements over [1] may also be achieved, at least in some cases. The examples in this correspondence also illustrate the gradual loss of performance from a "full-PDC" fuzzy controller to a "robust linear" one as uncertainty in the memberships increases.

\section{REFERENCES}

[1] H. K. Lam and F. H. F. Leung, "Stability analysis of fuzzy control systems subject to uncertain grades of membership," IEEE Trans. Syst., Man, Cybern. B, Cybern., vol. 35, no. 6, pp. 1322-1325, Dec. 2005.

[2] T. Takagi and M. Sugeno, "Fuzzy identification of systems and its applications to modelling and control," IEEE Trans. Syst., Man, Cybern., vol. SMC-15, no. 1, pp. 116-132, Feb. 1985.

[3] K. Tanaka and H. O. Wang, Fuzzy Control Systems Design and Analysis. New York: Wiley, 2001.

[4] X. Liu and Q. Zhang, "New approaches to $H_{\infty}$ controller designs based on fuzzy observers for T-S fuzzy systems via LMI," Automatica, vol. 39, no. 9, pp. 1571-1582, Sep. 2003.

[5] F. H. Hsiao, J. D. Hwang, C. W. Chen, and Z. R. Tsai, "Robust stabilization of nonlinear multiple time-delay large-scale systems via decentralized fuzzy control," IEEE Trans. Fuzzy Syst., vol. 13, no. 1, pp. 152-163, Feb. 2005.

[6] T. M. Guerra, A. Kruszewski, L. Vermeiren, and H. Tirmant, "Conditions of output stabilization for nonlinear models in the Takagi-Sugeno's form," Fuzzy Sets Syst., vol. 157, no. 9, pp. 1248-1259, May 2006.

[7] A. Sala and C. Ariño, "Asymptotically necessary and sufficient conditions for stability and performance in fuzzy control: Applications of Polya's theorem," Fuzzy Sets Syst., vol. 158, no. 4, pp. 2671-2686, Dec. 2007.

[8] J. Bondia, A. Sala, J. Picó, and M. A. Sainz, "Controller design under fuzzy pole-placement specifications: An interval arithmetic approach," IEEE Trans. Fuzzy Syst., vol. 14, no. 6, pp. 822-836, Dec. 2006.

[9] A. Sala, T. M. Guerra, and R. Babuška, "Perspectives of fuzzy systems and control," Fuzzy Sets Syst., vol. 156, no. 3, pp. 432-444, Dec. 2005.

[10] G. Feng, "A survey on analysis and design of model-based fuzzy control systems," IEEE Trans. Fuzzy Syst., vol. 14, no. 5, pp. 676-697, Oct. 2006.

[11] H. K. Lam and F. H. F. Leung, "Fuzzy controller with stability and performance rules for nonlinear systems," Fuzzy Sets Syst., vol. 158, no. 2, pp. 147-163, Jan. 2007.

[12] T. M. Guerra and L. Vermeiren, "LMI-based relaxed nonquadratic stabilization conditions for nonlinear systems in the Takagi-Sugeno's form," Automatica, vol. 40, no. 5, pp. 823-829, 2004.

[13] A. Sala and C. Ariño, "Relaxed stability and performance conditions for Takagi-Sugeno fuzzy systems with knowledge on membership function overlap," IEEE Trans. Syst., Man, Cybern. B, Cybern., vol. 37, no. 3, pp. 727-732, Jun. 2007.

[14] C. Ariño and A. Sala, "Relaxed LMI conditions for closed loop fuzzy systems with tensor product structure," Eng. Appl. Artif. Intell., vol. 20, no. 8, pp. 1036-1046, 2007.

[15] S. Boyd, L. El Ghaoui, E. Feron, and V. Balakrishnan, Linear Matrix Inequalities in System and Control Theory. Philadelphia, PA: SIAM, 1994.

[16] H. D. Tuan, P. Apkarian, T. Narikiyo, and Y. Yamamoto, "Parameterized linear matrix inequality techniques in fuzzy control system design," IEEE Trans. Fuzzy Syst., vol. 9, no. 2, pp. 324-332, Apr. 2001.

[17] E. Kim and H. Lee, "New approaches to relaxed quadratic stability condition of fuzzy control systems," IEEE Trans. Fuzzy Syst., vol. 8, no. 5, pp. 523-534, Oct. 2000. 